Non-linear modeling of active biohybrid materials

\author{
C. Paetsch
}

A. Dorfmann 



\title{
Non-linear modeling of active biohybrid materials
}

\author{
C. Paetsch ${ }^{\mathrm{a}}$, A. Dorfmann ${ }^{\mathrm{a}, \mathrm{b}, *}$ \\ ${ }^{a}$ Department of Civil and Environmental Engineering, Tufts University, \\ Medford, MA 02155, USA \\ ${ }^{b}$ Department of Biomedical Engineering, Tufts University, Medford, MA 02155, USA
}

\begin{abstract}
Recent advances in engineered muscle tissue attached to a synthetic substrate motivates the development of appropriate constitutive and numerical models. Applications of active materials can be expanded by using robust, non-mammalian muscle cells, such as those of Manduca sexta. In this study, we propose a model to assist in the analysis of biohybrid constructs by generalizing a recently proposed constitutive law for Manduca muscle tissue. The continuum model accounts (i) for the stimulation of muscle fibers by introducing multiple stress-free reference configurations for the active and passive states and (ii) for the hysteretic response by specifying a pseudo-elastic energy function. A simple example representing uniaxial loading-unloading is used to validate and verify the characteristics of the model. Then, based on experimental data of muscular thin films, a more complex case shows the qualitative potential of Manduca muscle tissue in active biohybrid constructs.
\end{abstract}

Keywords: Finite deformations; active tension; pseudo-elasticity, transverse isotropy; muscle tissue

\section{Introduction}

In nature, muscles perform as efficient, robust actuators capable of large non-linear elastic deformations. The internal structure is hierarchical with fibers embedded in a matrix of connective tissue, which transition from passive to active states and vice versa (Dorfmann et al., 2007). Additionally,

\footnotetext{
${ }^{*}$ Corresponding author

Email address: Luis.Dorfmann@tufts.edu (A. Dorfmann)

$U R L:$ http://ase.tufts.edu/msml/ (A. Dorfmann)
} 
their ability to generate tension and provide positive mechanical work under an action potential (Woods et al., 2008) make muscles unique amongst biological tissue and attractive for use in engineered devices. Developments in tissue engineering have made it possible to fabricate constructs of muscle cells which may be combined with an artificial substrate, such as polydimethylsiloxane (PDMS), to create an active biohybrid material. These innovative materials have been used in various demonstration projects to function as bioactuators. Cultured muscle tissues are advantageous over engineered actuators because of their ability to vary in size from the micro to macro scale (Feinberg et al., 2007; Hollerbach et al., 1991) and achieve life-like movements (Herr and Dennis, 2004; Nawroth et al., 2012). Furthermore, muscle tissue may be directly actuated with the application of a simple electrical stimulus (Akiyama et al., 2010) and can take advantage of dense energy sources, such as glucose and lipids (Tanaka et al., 2007). Potentially, hindering further development of bioactuators is the time and cost associated with culturing cells for prototype devices. These obstacles may be mitigated by using mathematical models to first evaluate potential designs.

An early application of bioactuation was demonstrated using frog skeletal muscles for a swimming robot (Herr and Dennis, 2004). The robot used explanted muscle tissue, which did not allow for flexibility in actuator design. To overcome this limitation, cultured muscle tissue on a polymer substrate have recently been used. This approach has been applied using mammalian cardiomyocytes to create proof-of-concept devices including a force transducer (Xi et al., 2005), fluidic pump (Tanaka et al., 2007), gripper (Feinberg et al., 2007), biomimetic propulsion (Nawroth et al., 2012) and potential drug delivery device (Fujita et al., 2011). An advantageous characteristic of mammalian cardiomyocytes' is their ability to spontaneously contract without external input. However, they require well-controlled environmental conditions, which limits the possible applications (Akiyama et al., 2010; Baryshyan et al., 2012). Other studies have focused on insect muscle cells, which can function under a wider range of conditions for a longer period of time. For example, cultured lepidopteran dorsal vessel tissues have demonstrated the ability to displace a micropillar array (Akiyama et al., 2010) and could potentially be used as an actuator. The cultured myoblasts of the tobacco hornworm, Manduca sexta, provide yet another alternative, as a recent study has demonstrated (Baryshyan et al., 2012). Potential advantages of Manduca tissue include a simpler muscle fiber structure and the ability for oxygen to be supplied to the tissue via diffusion, eliminating the need for a vascular 
system. These attributes make it an attractive choice for its application in bioactuators and will be addressed in this study.

Concurrent with the success of bioactuator prototyping is the need to develop mathematical models of biohybrid materials, which may assist in the design of devices. Modeling the continuous transition between stimulation states is of particular significance to evaluate actuator performance. The creation of active tension in a muscle is commonly accounted for by introducing an additional term in the stress formulation. Models following this approach have been developed for myocardium and adapted for skeletal muscles (Nash and Hunter, 2000; Nordsetten et al., 2011; Pathmanathan et al., 2010; Usyk et al., 2000; Whiteley et al., 2007). A prominent example of modeling active stress in skeletal muscle is the work by Hill (1938), where an active forcelength relationship is defined. An alternatively approach is based on the use of an augmented energy function, where an additive term is defined to account for the stimulated muscle fibers (Ehret et al., 2011; Ito et al., 2010; Odegard et al., 2008).

The method, known as active strain, utilizes the multiplicative decomposition of the strain tensor (Ambrosi and Pezzuto, 2012). Nardinocchi and Teresi (2007) model cardiac muscle as a hyperelastic material with an isotropic fiber distribution where the active deformation is assumed to be a physiologically-based constant parameter. In a more recent study, the model is expanded to include a relation between the active strain and the chemical potential, i.e. the calcium ion concentration in the muscle (Cherubini et al., 2008). Multiplicative decomposition of the deformation gradient is employed by Boel et al. (2009) and Shim et al. (2012) to model the changing reference configuration of cardiac muscle. The resulting model accounts for the response of both the active and passive fiber contributions while neglecting energy dissipation. For smooth muscles, Stålhand et al. (2011) propose a coupled chemo-mechanical free energy function to describe the behavior of active tissue accounting for an anisotropic fiber distribution. The straindependent contribution to the energy function is additively decomposed into passive and active parts, with the latter being dependent on the cross-bridge interaction.

In this paper, we expand the formulation used by Paetsch et al. (2012) to model the continuous transition of muscle tissue between passive and active states, assume the general case of compressible materials and simulate the complex movement of active biohybrid constructs. We begin in Section 2 with an overview of the basic equations required to model a transversely 
isotropic hyperelastic material. Next, we define the transient network theory using the notation of Rajagopal and Wineman (1992) and provide a brief review of pseudo-elasticity as it applies to muscle tissue. Section 3 contains the increments of kinematic and energy formulations necessary to derive the fourth-order elasticity tensor for a transversely isotropic material, with and without considering the effect of stress-softening. These general forms are specialized, in Section 3.3, to a specific constitutive law for a muscle transitioning between activation states. Uniaxial loading-unloading is used in Section 4 to verify and validate the characteristics of the model. We conclude with the simulation of a biohybrid gripper and show that cyclic contraction of muscle fibers induces coiling and uncoiling of a rectangular strip.

\section{Basic Equations}

Muscles possess a complex and hierarchical structure, with the active tension of the whole muscle generated by proteins interacting on the molecular scale (Lieber, 2002). Our approach does not aim to model the individual micro-constituents of muscle tissue. Instead, we attempt to capture the mechanical behavior using phenomenological models. In this section, we give an overview of the kinematic tensors and associated invariants, along with a general pseudo-elastic energy function and the related expression of stress.

\subsection{Kinematics}

To describe the conformational change of a deformable body we select an arbitrary chosen configuration as a reference, which we denote by $\mathcal{B}_{0}$. The location of any material particle in $\mathcal{B}_{0}$ is identified by its position vector $\mathbf{X}$ relative to some origin. Let $\mathcal{B}$ denote the configuration of the body obtained by a continuous transformation $\mathrm{x}=\boldsymbol{\chi}(\mathbf{X})$, where the vector function $\chi$ assigns to each point $\mathbf{X}$ a unique position $\mathbf{x}$ in $\mathcal{B}$ and vice versa. The deformation gradient tensor relative to the configuration $\mathcal{B}_{0}$ is defined by

$$
\mathbf{F}=\frac{\partial \boldsymbol{\chi}(\mathbf{X})}{\partial \mathbf{X}}=\frac{\partial \mathbf{x}}{\partial \mathbf{X}}
$$

with Cartesian components $F_{i \alpha}=\partial x_{i} / \partial X_{\alpha}$, where $i, \alpha \in\{1,2,3\}$. Roman indices are associated with the current configuration $\mathcal{B}$ and Greek indices with the reference configuration $\mathcal{B}_{0}$. We also use the standard notation $J$ to denote the volume ratio given by

$$
J=\operatorname{det} \mathbf{F}=\frac{\mathrm{d} v}{\mathrm{~d} V}>0
$$


where $\mathrm{d} v$ and $\mathrm{d} V$ denote corresponding volume elements in $\mathcal{B}$ and $\mathcal{B}_{0}$, respectively. Frequently, deformations of many biological material are said to be isochoric such that $J=1$ (volume-preserving), which is of course an idealization. In the following we relax this constraint and assume the general case of compressible materials with $J \neq 1$. Following the developments given by Flory $(1961)$ and Ogden $(1976,1978)$ we adopt the multiplicative decomposition of the total deformation into volumetric and isochoric parts expressed by

$$
\mathbf{F}=\left(J^{1 / 3}\right) \overline{\mathbf{F}},
$$

where $\overline{\mathbf{F}}$ is the isochoric portion of the deformation with $\operatorname{det} \overline{\mathbf{F}}=1$. The right and left Cauchy-Green tensors are respectively given by

$$
\mathbf{C}=\mathbf{F}^{\mathrm{T}} \mathbf{F}, \quad \mathbf{B}=\mathbf{F F}^{\mathrm{T}},
$$

and, using (3), the corresponding isochoric counterparts are

$$
\overline{\mathbf{C}}=\overline{\mathbf{F}}^{\mathrm{T}} \overline{\mathbf{F}}=\left(J^{-2 / 3}\right) \mathbf{C}, \quad \overline{\mathbf{B}}=\overline{\mathbf{F}} \overline{\mathbf{F}}^{\mathrm{T}}=\left(J^{-2 / 3}\right) \mathbf{B} .
$$

The principle invariants of $\mathbf{C}$ (equivalently B) are denoted by $I_{1}, I_{2}, I_{3}$ and defined by

$$
I_{1}=\operatorname{tr} \mathbf{C}, \quad I_{2}=\frac{1}{2}\left[(\operatorname{tr} \mathbf{C})^{2}-\operatorname{tr}\left(\mathbf{C}^{2}\right)\right], \quad I_{3}=\operatorname{det} \mathbf{C}=J^{2},
$$

where tr denotes the trace of the second-order tensor. We may also write the corresponding volume-preserving invariants, i.e. the principle invariants of $\overline{\mathbf{C}}$ (equivalently $\overline{\mathbf{B}}$ ), as

$$
\bar{I}_{1}=\operatorname{tr} \overline{\mathbf{C}}=J^{-2 / 3} I_{1}, \quad \bar{I}_{2}=\frac{1}{2}\left[(\operatorname{tr} \overline{\mathbf{C}})^{2}-\operatorname{tr}\left(\overline{\mathbf{C}}^{2}\right)\right]=J^{-4 / 3} I_{2}, \quad \bar{I}_{3} \equiv 1 .
$$

Here, muscle is considered to be a fiber-reinforced material with a preferred direction given by its internal structure, namely fascicles comprised of myofibril bundles. The direction of the homogeneous distribution of fibers is locally specified by the unit vector $\mathbf{M}$ in the reference configuration $\mathcal{B}_{0}$. Following the work by Spencer (1971) two additional invariants must be defined to characterize 'transversely isotropic materials'. These are

$$
I_{4}=\mathbf{M} \cdot \mathbf{C M}, \quad I_{5}=\mathbf{M} \cdot \mathbf{C}^{2} \mathbf{M},
$$


where the invariant $I_{4}$ is the square of the stretch in the fiber direction M. No similar physical interpretation exists for $I_{5}$. The isochoric invariant counterparts of $I_{4}, I_{5}$ are given by

$$
\bar{I}_{4}=\mathbf{M} \cdot \overline{\mathbf{C}} \mathbf{M}=\left(J^{-2 / 3}\right) I_{4}, \quad \bar{I}_{5}=\mathbf{M} \cdot \overline{\mathbf{C}}^{2} \mathbf{M}=\left(J^{-4 / 3}\right) I_{5} .
$$

For details of the kinematics of isotropic and transversely isotropic materials, we refer to, for example, Ogden (2001).

\subsection{Hyperelastic Material}

We use the theory of hyperelasticity to characterize the elastic response of muscle tissue. The general theory is based on the use of an elastic strain energy function $W(\mathbf{F})$, defined per unit volume in the reference configuration $\mathcal{B}_{0}$. From the energy balance equation we find that the nominal stress $\mathbf{S}$ is given by

$$
\mathbf{S}=\frac{\partial W}{\partial \mathbf{F}}, \quad S_{\alpha i}=\frac{\partial W}{\partial F_{i \alpha}}
$$

where we identified our convention for the order of indices in differentiation with respect to a nonsymmetric second-order tensor.

Integration over a surface and the use of Nanson's formula (Ogden, 1997) gives the Cauchy stress tensor

$$
\boldsymbol{\sigma}=J^{-1} \mathbf{F} \frac{\partial W}{\partial \mathbf{F}}, \quad \sigma_{i j}=J^{-1} F_{i \alpha} \frac{\partial W}{\partial F_{j \alpha}} .
$$

For an isotropic hyperelastic material, the function $W$ depends on only three scalar measures of deformation and we use the principal invariants $I_{1}, I_{2}$ and $I_{3}$ specified in (6). Then, we can express the nominal stress tensor in the form

$$
\mathbf{S}=\frac{\partial W}{\partial \mathbf{F}}=\sum_{i=1}^{3} \frac{\partial W}{\partial I_{i}} \frac{\partial I_{i}}{\partial \mathbf{F}},
$$

where, for simplicity, we used the same notation $W$ to denote two functions $W(\mathbf{F})$ and $W\left(I_{1}, I_{2}, I_{3}\right)$.

For a transversely isotropic material, the strain energy function must be augmented to include the dependence on the two additional invariants $I_{4}$ and $I_{5}$, given by (8). To represent the strain energy as a function of the five invariants we use the notation

$$
W=W\left(I_{1}, I_{2}, I_{3}, I_{4}, I_{5}\right) .
$$


Following Bose and Dorfmann (2009); Prot and Skallerud (2009), we now specialize (13) by additively decomposing $W$ into volumetric and deviatoric parts

$$
W=\bar{W}\left(\bar{I}_{1}, \bar{I}_{2}, \bar{I}_{4}, \bar{I}_{5}\right)+U(J)
$$

where $\bar{W}\left(\bar{I}_{1}, \bar{I}_{2}, \bar{I}_{4}, \bar{I}_{5}\right)$ is the volume-preserving contribution and $U(J)$ is the volumetric portion. It is noted by Federico and Gasser (2010) that the decoupled strain energy formulation (14) requires a coupling term when large changes in volume occur.

Substitution of expression (14) into (10) gives additive components of the nominal stress tensor

$$
\mathbf{S}=\frac{\partial \bar{W}}{\partial \mathbf{F}}+\frac{\partial U}{\partial \mathbf{F}}=\overline{\mathbf{S}}+\mathbf{S}_{\mathrm{vol}}
$$

where the first term on the right hand side represents the isochoric (or deviatoric) contribution, denoted $\overline{\mathbf{S}}$, and the second term is the volumetric (or hydrostatic) contribution, denoted $\mathbf{S}_{\mathrm{vol}}$. Evaluation of equation (15) requires the derivatives of the isochoric strain invariants with respect to $\mathbf{F}$. For reference these are summarized here in the form

$$
\begin{aligned}
& \frac{\partial \bar{I}_{1}}{\partial \mathbf{F}}=2 J^{-1 / 3}\left[\overline{\mathbf{F}}^{\mathrm{T}}-\frac{1}{3} \bar{I}_{1} \overline{\mathbf{F}}^{-1}\right], \\
& \frac{\partial \bar{I}_{2}}{\partial \mathbf{F}}=2 J^{-1 / 3}\left[\bar{I}_{1} \overline{\mathbf{F}}^{\mathrm{T}}-\overline{\mathbf{F}}^{\mathrm{T}} \overline{\mathbf{F}} \overline{\mathbf{F}}^{\mathrm{T}}-\frac{2}{3} \bar{I}_{2} \overline{\mathbf{F}}^{-1}\right], \\
& \frac{\partial \bar{I}_{4}}{\partial \mathbf{F}}=2 J^{-1 / 3}\left[\mathbf{M} \otimes \overline{\mathbf{F}} \mathbf{M}-\frac{1}{3} \bar{I}_{4} \overline{\mathbf{F}}^{-1}\right], \\
& \frac{\partial \bar{I}_{5}}{\partial \mathbf{F}}=2 J^{-1 / 3}\left[\mathbf{M} \otimes \overline{\mathbf{F}} \overline{\mathbf{C}} \mathbf{M}+\overline{\mathbf{C}} \mathbf{M} \otimes \overline{\mathbf{F}} \mathbf{M}-\frac{2}{3} \bar{I}_{5} \overline{\mathbf{F}}^{-1}\right],
\end{aligned}
$$

where $\partial J / \partial \mathbf{F}=J \mathbf{F}^{-1}$. Applying the chain rule to equation (15) and substituting (16) through (19), we obtain the explicit expressions for the isochoric and volumetric components of the nominal stress tensor as

$$
\begin{aligned}
\overline{\mathbf{S}} & =2 J^{-1 / 3}\left[\bar{W}_{1}\left(\overline{\mathbf{F}}^{\mathrm{T}}-\frac{1}{3} \bar{I}_{1} \overline{\mathbf{F}}^{-1}\right)+\bar{W}_{2}\left(\bar{I}_{1} \overline{\mathbf{F}}^{\mathrm{T}}-\overline{\mathbf{F}}^{\mathrm{T}} \overline{\mathbf{F}} \overline{\mathbf{F}}^{\mathrm{T}}-\frac{2}{3} \bar{I}_{2} \overline{\mathbf{F}}^{-1}\right)\right. \\
& \left.+\bar{W}_{4}\left(\mathbf{M} \otimes \overline{\mathbf{F}} \mathbf{M}-\frac{1}{3} \bar{I}_{4} \overline{\mathbf{F}}^{-1}\right)+\bar{W}_{5}\left(\mathbf{M} \otimes \overline{\mathbf{F}} \overline{\mathbf{C}} \mathbf{M}+\overline{\mathbf{C}} \mathbf{M} \otimes \overline{\mathbf{F}} \mathbf{M}-\frac{2}{3} \bar{I}_{5} \overline{\mathbf{F}}^{-1}\right)\right], \\
\mathbf{S}_{\mathrm{vol}} & =J \frac{\mathrm{d} U}{\mathrm{~d} J} \mathbf{F}^{-1},
\end{aligned}
$$


where we used the notation $\bar{W}_{i}=\partial \bar{W} / \partial \bar{I}_{i}$ for $i=1,2,4,5$. Using equation (11), with the total nominal stress $\mathbf{S}=\overline{\mathbf{S}}+\mathbf{S}_{\mathrm{vol}}$, gives the isochoric and volumetric parts to the total Cauchy stress tensor $\boldsymbol{\sigma}$. These are denoted respectively by $\overline{\boldsymbol{\sigma}}$ and $\boldsymbol{\sigma}_{\mathrm{vol}}$ and are given by

$$
\begin{aligned}
\overline{\boldsymbol{\sigma}} & =2 J^{-1}\left[\bar{W}_{1}\left(\overline{\mathbf{B}}-\frac{1}{3} \bar{I}_{1} \mathbf{I}\right)+\bar{W}_{2}\left(\bar{I}_{1} \overline{\mathbf{B}}-\overline{\mathbf{B}}^{2}-\frac{2}{3} \bar{I}_{2} \mathbf{I}\right)\right. \\
& \left.+\bar{W}_{4}\left(\overline{\mathbf{m}} \otimes \overline{\mathbf{m}}-\frac{1}{3} \bar{I}_{4} \mathbf{I}\right)+\bar{W}_{5}\left(\overline{\mathbf{m}} \otimes \overline{\mathbf{B}} \overline{\mathbf{m}}+\overline{\mathbf{B}} \overline{\mathbf{m}} \otimes \overline{\mathbf{m}}-\frac{2}{3} \bar{I}_{5} \mathbf{I}\right)\right], \\
\boldsymbol{\sigma}_{\mathrm{vol}} & =\frac{\mathrm{d} U}{\mathrm{~d} J} \mathbf{I}
\end{aligned}
$$

where $\overline{\mathbf{m}}=\overline{\mathbf{F}} \mathbf{M}$.

Expressions (22) and (23) can be written more concisely by introducing the deviatoric operator defined as $\operatorname{dev}(\mathbf{A})=\mathbb{P}: \mathbf{A}$, where the fourth-order projection tensor $\mathbb{P}$ has components $\mathbb{P}_{i j k l}=\delta_{i k} \delta_{j l}-(1 / 3) \delta_{i j} \delta_{k l}$ and $\mathbf{A}$ is a second-order symmetric tensor. The component form of $\mathbb{P}: \mathbf{A}$ is $\mathbb{P}_{i j k l} A_{k l}$. The total Cauchy stress $\boldsymbol{\sigma}=\overline{\boldsymbol{\sigma}}+\boldsymbol{\sigma}_{\mathrm{vol}}$ can then be written in a more compact form

$$
\begin{aligned}
\boldsymbol{\sigma} & =2 J^{-1}\left[\bar{W}_{1} \operatorname{dev}(\overline{\mathbf{B}})+\bar{W}_{2} \operatorname{dev}\left(\bar{I}_{1} \overline{\mathbf{B}}-\overline{\mathbf{B}}^{2}\right)+\bar{W}_{4} \operatorname{dev}(\overline{\mathbf{m}} \otimes \overline{\mathbf{m}})\right. \\
& \left.+\bar{W}_{5} \operatorname{dev}(\overline{\mathbf{m}} \otimes \overline{\mathbf{B}} \overline{\mathbf{m}}+\overline{\mathbf{B}} \overline{\mathbf{m}} \otimes \overline{\mathbf{m}})\right]+\frac{\mathrm{d} U}{\mathrm{~d} J} \mathbf{I}
\end{aligned}
$$

\subsection{Active fibers}

A muscle fiber contracts during activation if no mechanical load is applied. Alternatively, if a stimulus is applied while the muscle is held at a constant length it develops a contractile force. Understanding the basic physiology of muscle contraction is essential to support modeling efforts. To that end, we describe the muscle fibers as multinucleate cells consisting of partially overlapping strings of thick (myocin protein) and thin (actin protein) filaments (Craig and Padrón, 2004; Perry, 1996). Muscle contraction is initiated by an action potential transmitted by the motor neuron, resulting in the release of calcium ions $\left(\mathrm{Ca}^{+}\right)$. The increased concentration of $\mathrm{Ca}^{+}$leads to an increase in the actin-myocin interaction. Specifically, cross-bridging between actin and myosin begin with the head of myosin molecules attached to the actin filament. While still connected, the myosin head flexes and rotates, thereby sliding the actin molecule along the myosin filament. Afterwards, the myosin detaches from the actin molecule, freeing the myosin head to return to the 
unflexed position and to reconnect again with the actin molecule further up the filament. During activation, the entire process is repeated continuously, inducing contractile properties to the muscle fiber. When the motor neuron stops stimulating the muscle, the concentration of calcium ions drops and interactions are inhibited, resulting in the muscle returning to its relaxed state (Nossal and Lecar, 1991). The attachment and detachment of myosin from the actin molecules is similar to the breakage and reformation of cross-links in polymeric materials, the latter of which is described by the theory of transient networks (Demirkoparan et al., 2009; Rajagopal and Wineman, 1992). Following the developments presented by Paetsch et al. (2012); Cherubini et al. (2008) and Nardinocchi and Teresi (2007) we use a model based on the transient network theory to describe the mechanical behavior of muscle fibers.

Let $s$ denote a parameter which uniquely identifies the set of all possible individual configurations $\mathcal{B}(s)$ of the body. Following notation used by Horgan et al. (2004) and Rajagopal1992 we take $s=0$ to define the initial, passive state of the muscle in the natural (stress-free) configuration $\mathcal{B}(0)=\mathcal{B}_{0}$. The muscle, in the absence of any boundary traction, is now subjected to an external stimulus that induces a deformation in the material. Let the corresponding state be identified by $s=\hat{s}$ and the configuration occupied by the material by $\mathcal{B}(\hat{s})=\hat{\mathcal{B}}$. Suppose that the activated muscle is now subjected to mechanically loads and transitions to the current state identified by the parameter $s$ with configuration denoted by $\mathcal{B}(s)=\mathcal{B}$. For further information on multiple natural configurations and their application to modeling muscles, see Paetsch et al. (2012).

Assume the transition from $\mathcal{B}_{0}$ to $\hat{\mathcal{B}}$, induced by an electro-physiological stimulus, occurs at constant volume in the absence of any mechanical load. The resulting isochoric deformation is defined by the gradient $\overline{\mathbf{F}}(\hat{s}, 0)$, which we write more concisely as $\overline{\mathbf{F}}_{0}$. The corresponding right and left CauchyGreen deformation tensors, defined in Subsection 2.1, have the forms

$$
\overline{\mathbf{C}}_{0}=\overline{\mathbf{F}}_{0}^{\mathrm{T}} \overline{\mathbf{F}}_{0}, \quad \overline{\mathbf{B}}_{0}=\overline{\mathbf{F}}_{0} \overline{\mathbf{F}}_{0}^{\mathrm{T}} .
$$

The corresponding invariants related to the fiber direction are

$$
\bar{I}_{4_{0}}=\mathbf{M} \cdot \overline{\mathbf{C}}_{0} \mathbf{M}, \quad \bar{I}_{5_{0}}=\mathbf{M} \cdot \overline{\mathbf{C}}_{0}^{2} \mathbf{M},
$$

where the unit vector $\mathbf{M}$ defines the preferred direction in $\mathcal{B}_{0}$. We now introduce the multiplicative decomposition of the isochoric deformation gradient 
$\overline{\mathbf{F}}(s, 0)$ as

$$
\overline{\mathbf{F}}(s, 0)=\overline{\mathbf{F}}(s, \hat{s}) \overline{\mathbf{F}}(\hat{s}, 0),
$$

where $\overline{\mathbf{F}}(s, \hat{s})$ is the gradient resulting from the application of external loads. We introduce, for convenience, the abbreviations $\overline{\mathbf{F}}=\overline{\mathbf{F}}(s, 0)$ and $\hat{\overline{\mathbf{F}}}=\overline{\mathbf{F}}(s, \hat{s})$ and write (27) more concisely as

$$
\overline{\mathbf{F}}=\hat{\overline{\mathbf{F}}} \overline{\mathbf{F}}_{0} .
$$

We use the decomposition (28) to define the elastic deformation of active muscle fibers in the current configuration $\mathcal{B}$ relative to the intermediate configuration $\hat{\mathcal{B}}$ given by $\hat{\overline{\mathbf{F}}}=\overline{\mathbf{F}} \overline{\mathbf{F}}_{0}^{-1}$. The corresponding right and left Cauchy-Green tensors, denoted respectively by $\hat{\overline{\mathbf{C}}}$ and $\hat{\overline{\mathbf{B}}}$, are defined as

$$
\hat{\overline{\mathbf{C}}}=\overline{\mathbf{F}}_{0}^{-\mathrm{T}} \overline{\mathbf{C}} \overline{\mathbf{F}}_{0}^{-1}, \quad \hat{\overline{\mathbf{B}}}=\overline{\mathbf{F}} \overline{\mathbf{C}}_{0}^{-1} \overline{\mathbf{F}}^{\mathrm{T}},
$$

with the corresponding invariants given by

$$
\hat{\bar{I}}_{4}=\hat{\mathbf{M}} \cdot \hat{\overline{\mathbf{C}}} \hat{\mathbf{M}}, \quad \hat{\bar{I}}_{5}=\hat{\mathbf{M}} \cdot \hat{\overline{\mathbf{C}}}^{2} \hat{\mathbf{M}}
$$

where the unit vector $\hat{\mathbf{M}}$ specifies the fiber direction in the intermediate configuration and is given by $\hat{\mathbf{M}}=\bar{I}_{4_{0}}^{-1 / 2} \overline{\mathbf{F}}_{0} \mathbf{M}$. Following the relation in (28), the invariant $\bar{I}_{4}$ may be decomposed as

$$
\bar{I}_{4}=\hat{\bar{I}}_{4} \bar{I}_{40}
$$

with no similar decomposition possible for $\hat{\bar{I}}_{5}$.

In general, $\overline{\mathbf{F}}_{0}$ may depend on $\mathbf{F}$. However, for simplicity, we assume that $\overline{\mathbf{F}}_{0}$ depends only on the internal structure of the muscle's sarcomere. The derivation of the deviatoric components of the nominal stress (20) or of the Cauchy stress $(22)$, requires the derivatives of $\hat{\bar{I}}_{4}$ and $\hat{\bar{I}}_{5}$ with respect to $\mathbf{F}$. These are given by

$$
\begin{aligned}
\frac{\partial \hat{\bar{I}}_{4}}{\partial \mathbf{F}} & =\frac{\partial\left(\bar{I}_{4} \bar{I}_{4_{0}}^{-1}\right)}{\partial \mathbf{F}}=2 J^{-1 / 3} \bar{I}_{4_{0}}^{-1}\left[\mathbf{M} \otimes \overline{\mathbf{F}} \mathbf{M}-\frac{1}{3} \bar{I}_{4} \overline{\mathbf{F}}^{-1}\right] \\
\frac{\partial \hat{\bar{I}}_{5}}{\partial \mathbf{F}} & =2 J^{-1 / 3} \bar{I}_{4_{0}}^{-1}\left[\mathbf{M} \otimes \overline{\mathbf{F}} \overline{\mathbf{C}}_{0}^{-1} \overline{\mathbf{C}} \mathbf{M}+\overline{\mathbf{C}}_{0}^{-1} \overline{\mathbf{C}} \mathbf{M} \otimes \overline{\mathbf{F}} \mathbf{M}\right. \\
& \left.-\frac{2}{3}\left(\mathbf{M} \cdot \overline{\mathbf{C}} \overline{\mathbf{C}}_{0}^{-1} \overline{\mathbf{C}} \mathbf{M}\right) \overline{\mathbf{F}}^{-1}\right] .
\end{aligned}
$$




\subsection{Pseudo-elasticity}

Pseudo-elasticity is defined as a material behavior where the stress during unloading is significantly less than during loading for the same stretch, even though the body may return to its original reference configuration. The theoretical framework, developed by Ogden and Roxburgh (1999), has been used by Dorfmann and Ogden (2004) to model stress-softening of particle reinforced elastomers during the first few loading cycles. The theory has been adapted to model the hysteretic behavior during loading-partial unloading and reloading of rubberlike solids by Dorfmann and Ogden (2003). A similar stress-softening phenomenon also characterizes many biological materials, such as muscle (Dorfmann et al., 2007, 2008; Paetsch et al., 2012; Woods et al., 2008), artery and skin tissue (Fung, 1967, 1980).

The theory is based on the condition that the elastic strain energy function $W(\mathbf{F})$ can be modified by incorporating a scalar parameter $\eta$ such that

$$
W=W(\mathbf{F}, \eta)
$$

where $W(\mathbf{F}, \eta)$ is referred to as the pseudo-energy function. The inclusion of $\eta$ provides a means of changing the form of the energy function during the deformation process. We assume that during loading the variable $\eta$ is inactive with constant value of unity, which leaves the strain energy function unaltered and we rewrite equation (34) as

$$
W_{0}(\mathbf{F})=W(\mathbf{F}, 1)
$$

where the subscript 0 is used to indicate that $\eta$ is inactive, a notation which will be utilized for quantities dependent on the derivatives of $W_{0}$ as well. During loading the nominal stress, defined by (10), has the form

$$
\mathbf{S}_{0}=\frac{\partial W_{0}}{\partial \mathbf{F}}(\mathbf{F})
$$

If $\eta$ is active we take it to depend on $\mathbf{F}$, and using (34) the nominal stress is given by

$$
\mathbf{S}=\frac{\partial W}{\partial \mathbf{F}}(\mathbf{F}, \eta)+\frac{\partial W}{\partial \eta}(\mathbf{F}, \eta) \frac{\partial \eta}{\partial \mathbf{F}} .
$$

This equation simplifies when the relation

$$
\frac{\partial W}{\partial \eta}(\mathbf{F}, \eta)=0
$$


holds, which is assumed in the following. The variable $\eta$ is then implicitly defined in terms of $\mathbf{F}$, such that

$$
\eta=\eta_{e}(\mathbf{F})
$$

Using (39) in (34) defines a reduced energy function $w$ given by

$$
w(\mathbf{F})=W\left(\mathbf{F}, \eta_{e}(\mathbf{F})\right),
$$

which depends on $\mathbf{F}$ only. The nominal stress is then given by the relation

$$
\mathbf{S}=\frac{\partial W}{\partial \mathbf{F}}(\mathbf{F}, \eta)=\frac{\partial w}{\partial \mathbf{F}}(\mathbf{F}),
$$

whether or not $\eta$ is active. When $\eta$ is turned on, its value is given by evaluating the right-hand side of equation (39).

The derivation of specific models within the general theory of pseudoelasticity is outside the scope of this study and we refer to Ogden and Roxburgh (1999) and Dorfmann and Ogden (2003, 2004) for details. Following the developments in muscle mechanics presented by Dorfmann et al. (2007) and Paetsch et al. (2012) we suggest the pseudo-energy function

$$
W(\mathbf{F}, \eta)=\eta W_{0}(\mathbf{F})+\phi(\eta),
$$

where the term $\phi(\eta)$ accounts for the energy dissipated during cyclic loadingunloading. In view of a decoupled strain energy formulation $W(\mathbf{F})=\bar{W}(\overline{\mathbf{F}})+$ $U(J)$, we assume that stress-softening is attributed to the deviatoric component of the deformation. The pseudo-energy function (42) is then specialized to

$$
W(\mathbf{F}, \eta)=\eta \bar{W}_{0}(\overline{\mathbf{F}})+\phi(\eta)+U(J) .
$$

During loading, when $\eta$ is inactive and equal to unity, equation (43) reads $W(\mathbf{F}, 1)=\bar{W}_{0}(\overline{\mathbf{F}})+\phi(1)+U(J)$ and the condition $\phi(1)=0$ follows. The constraint specified by equation (38) and applied to (43) becomes

$$
\phi^{\prime}(\eta)=-\bar{W}_{0}(\overline{\mathbf{F}}),
$$

where the prime is used to indicate differentiation with respect to $\eta$. The nominal stress using the energy function (43) has the form

$$
\mathbf{S}=\eta \frac{\partial \bar{W}_{0}}{\partial \mathbf{F}}(\overline{\mathbf{F}})+J \frac{\mathrm{d} U}{\mathrm{~d} J} \mathbf{F}^{-1}=\eta \overline{\mathbf{S}}_{0}+\mathbf{S}_{\mathrm{vol}}
$$


where $\eta=1$ during loading and specified by (44) otherwise. Using relation (11) gives the Cauchy stress as

$$
\boldsymbol{\sigma}=\eta J^{-1} \mathbf{F} \frac{\partial \bar{W}_{0}}{\partial \mathbf{F}}(\overline{\mathbf{F}})+\frac{\mathrm{d} U}{\mathrm{~d} J} \mathbf{I}=\eta \overline{\boldsymbol{\sigma}}_{0}+\boldsymbol{\sigma}_{\mathrm{vol}},
$$

where $\overline{\mathbf{S}}_{0}$ and $\overline{\boldsymbol{\sigma}}_{0}$ denote the deviatoric portions of the nominal and Cauchy stress, respectively, without the effects of stress-softening.

In addition to the formulation of $W(\overline{\mathbf{F}}, \eta)$ we need to specify the form of $\eta$, which must decay monotonically from unity when unloading is initiated at any point along the loading paths. In order to satisfy the above requirements, we select the derivative of the dissipation function $\phi(\eta)$ such that

$$
-\phi^{\prime}(\eta)=m \tanh ^{-1}[r(\eta-1)]+\bar{W}_{\mathrm{m}},
$$

where $r$ and $m$ are material parameters and $\bar{W}_{\mathrm{m}}$ is the current maximum value of the isochoric strain energy achieved on the loading path when unloading is initiated. From (47) we obtain the explicit expression

$$
\eta=1-\frac{1}{r} \tanh \left[\frac{\bar{W}_{\mathrm{m}}-\bar{W}_{0}(\overline{\mathbf{F}})}{m}\right] .
$$

Suppose that at an arbitrary point during unloading the material begins to reload. When reloading is initiated we take $\eta=\eta_{1}$ and $\overline{\mathbf{F}}=\overline{\mathbf{F}}_{1}$, and the value of $\eta$ during reloading, denoted $\eta_{\mathrm{r}}$, is given by

$$
\eta_{\mathrm{r}}=\eta_{1}+\left(1-\eta_{1}\right) \tanh \left[\frac{\bar{W}_{0}(\overline{\mathbf{F}})-\bar{W}_{1}}{a}\right],
$$

where $a$ is a material parameter and $\bar{W}_{1}$ is the isochoric strain energy when reloading is initiated, i.e. $\bar{W}_{1}=\bar{W}_{0}\left(\overline{\mathbf{F}}_{1}\right)$. If the material begins to unload again before the primary loading curve is reached with $\overline{\mathbf{F}}=\overline{\mathbf{F}}_{\text {ru }}$ and with $\eta=\eta_{\mathrm{ru}}$, we take the value of $\eta$ during the second unloading, denoted $\eta_{\mathrm{u}}$, to be

$$
\eta_{\mathrm{u}}=\eta_{\mathrm{ru}}-\eta_{\mathrm{ru}} \tanh \left[\frac{\bar{W}_{\mathrm{ru}}-\bar{W}_{0}(\overline{\mathbf{F}})}{b}\right],
$$

where $b$ is an additional material parameter and $\bar{W}_{\text {ru }}$ is the maximum isochoric strain energy obtained during reloading, i.e. $\bar{W}_{\mathrm{ru}}=\bar{W}_{0}\left(\overline{\mathbf{F}}_{\mathrm{ru}}\right)$. For a detailed presentation of a pseudo-elastic model for loading, partial unloading and reloading we refer to Dorfmann and Ogden (2003). 


\section{Numerical solution}

Muscles, like many biological systems, have an inherently irregular geometry and often experience complex loads, making analytical solutions difficult. Exact solutions are possible only for a limited number of problems restricting the use of a constitutive model to simple geometries and boundary conditions. In this section we develop the tensor quantities required for implementing a nearly incompressible, transversely isotropic, pseudo-elastic material in the finite element software Abaqus (Simulia, 2009). We first derive the necessary tensor quantities without including the effect of stress-softening, followed by the formulation of the same quantities in the context of pseudo-elasticity.

We identify a small virtual incremental displacement superimposed on $\boldsymbol{\chi}(\mathbf{X})$, which we denote by $\delta \mathbf{u}$. Through the transformation $\mathbf{x}=\boldsymbol{\chi}(\mathbf{X})$ we define $\delta \mathbf{u}$ as an Eulerian quantity and write

$$
\delta \mathbf{F}=\delta \mathbf{l} \mathbf{F},
$$

where $\delta \mathbf{l}$ is the virtual displacement gradient, which has the Cartesian components

$$
\delta l_{i j}=\frac{\partial \delta u_{i}}{\partial x_{j}} .
$$

This can be decomposed into the sum of a symmetric and an antisymmetric part as

$$
\delta \mathbf{l}=\delta \mathbf{d}+\delta \mathbf{w},
$$

where $\delta \mathbf{d}$ is the first variation of the rate-of-deformation and $\delta \mathbf{w}$ the first variation of the continuum spin. These are give by

$$
\delta \mathbf{d}=\frac{1}{2}\left(\delta \mathbf{l}+\delta \mathbf{l}^{\mathrm{T}}\right), \quad \delta \mathbf{w}=\frac{1}{2}\left(\delta \mathbf{l}-\delta \mathbf{l}^{\mathrm{T}}\right),
$$

which are respectively symmetric and antisymmetric.

The numerical solution is based on a variational formulation of the boundaryvalue problem. For completeness, we recall the internal virtual work expression in the form

$$
\int_{\mathcal{B}} \boldsymbol{\sigma}: \delta \mathbf{l} \mathrm{d} v=\int_{\mathcal{B}_{0}} J \boldsymbol{\sigma}: \delta \mathbf{l} \mathrm{d} V
$$

where we used the relation $\mathrm{d} v=J \mathrm{~d} V$ to convert the integral from the current region $\mathcal{B}$ to the reference region $\mathcal{B}_{0}$. In equation (55) we also introduced the operator : defined, for a pair of second order tensors $\mathbf{A}$ and $\mathbf{B}$, as double 
contraction $A_{i j} B_{i j}$. Since the Cauchy stress is symmetric the integrand $\boldsymbol{\sigma}: \delta \mathbf{l}$ may also be written as $\boldsymbol{\sigma}: \delta \mathbf{d}$, where $\delta \mathbf{d}$ is the symmetric part of $\delta \mathbf{l}$ given by $(54)_{1}$.

We now consider a small incremental displacement superimposed on the configuration $\mathcal{B}$, which we denote by $\mathrm{d} \mathbf{u}$ and, for convenience, is treated as a function of $\mathbf{x}$. We then obtain the important connection

$$
\mathrm{d} \mathbf{F}=\mathrm{d} \mathbf{l} \mathbf{F}
$$

where $\mathrm{d} \mathbf{l}$ is the spatial gradient of $\mathrm{d} \mathbf{u}$, with components defined by $\mathrm{d} l_{i j}=$ $\partial \mathrm{d} u_{i} / \partial x_{j}$. Following the same argument as in (53), the incremental displacement gradient dl can be additively decomposed into symmetric and antisymmetric parts denoted $\mathrm{d} \mathbf{d}$ and $\mathrm{d} \mathbf{w}$, respectively.

The use of Newton's method to solve the non-linear equilibrium equations requires linearization of the internal virtual work expression, which is given by

$$
\mathrm{d} \delta W_{\text {int }}=\int_{\mathcal{B}_{0}}[\mathrm{~d}(J \boldsymbol{\sigma}): \delta \mathbf{d}+J \boldsymbol{\sigma}: \mathrm{d} \delta \mathbf{d}] \mathrm{d} V .
$$

where here and henceforth the operator $\mathrm{d}$ is used to denote the increment of the quantity concerned, see Holzapfel (2000) for a detailed discussion of the variational and incremental operators.

Constitutive equations in Abaqus must be given in rate form as a relation between the Kirchhoff stress rate, $\mathrm{d}(J \boldsymbol{\sigma})$, and the rate of deformation dd. Objectivity of the stress rate resulting from the evaluation of $\mathrm{d}(J \boldsymbol{\sigma})$ is not guaranteed and, using continuum elements, the Jaumann rate of the Kirchhoff stress is required. Using the notation defined by Prot and Skallerud (2009) we write the Jaumann rate of $J \boldsymbol{\sigma}$ as

$$
\begin{aligned}
\mathrm{d}^{\nabla J}(J \boldsymbol{\sigma}) & =\mathrm{d}(J \boldsymbol{\sigma})-J(\mathrm{~d} \mathbf{w} \boldsymbol{\sigma}-\boldsymbol{\sigma} \mathrm{d} \mathbf{w}), \\
& =\mathbb{C}^{\nabla J}: \mathrm{d} \mathbf{d},
\end{aligned}
$$

where the operator $\mathrm{d}^{\nabla J}$ is used to denote the increment determined from the Jaumann rate and $\mathbb{C}^{\nabla J}$ is the corresponding symmetric fourth-order elasticity tensor. By equating the right-hand sides of equations (58) and (59) and solving for $\mathrm{d}(J \boldsymbol{\sigma})$, we obtain

$$
\mathrm{d}(J \boldsymbol{\sigma})=J\left(J^{-1} \mathbb{C}^{\nabla J}: \mathrm{d} \mathbf{d}+\mathrm{d} \mathbf{w} \boldsymbol{\sigma}-\boldsymbol{\sigma} \mathrm{d} \mathbf{w}\right) .
$$


Abaqus (Simulia, 2009) requires that the fourth-order elasticity tensor be implemented as

$$
\mathbb{C}=J^{-1} \mathbb{C}^{\nabla J}
$$

and we refer to Section 4.6 of the Abaqus Theory Manual for a detailed presentation. The Kirchhoff stress rate of the isochoric and volumetric components of the Cauchy stress tensor are denoted by $\mathrm{d}(J \overline{\boldsymbol{\sigma}})$ and $\mathrm{d}\left(J \boldsymbol{\sigma}_{\mathrm{vol}}\right)$, respectively. Equation (60) is then additively decomposed as

$$
\mathrm{d}(J \overline{\boldsymbol{\sigma}})=J\left(J^{-1} \overline{\mathbb{C}}^{\nabla \mathrm{J}}: \mathrm{d} \mathbf{d}+\mathrm{d} \mathbf{w} \overline{\boldsymbol{\sigma}}-\overline{\boldsymbol{\sigma}} \mathrm{d} \mathbf{w}\right),
$$

and

$$
\mathrm{d}\left(J \boldsymbol{\sigma}_{\text {vol }}\right)=J\left(J^{-1} \mathbb{C}_{\text {vol }}^{\nabla \mathrm{J}}: \mathrm{d} \mathbf{d}\right),
$$

where $\overline{\mathbb{C}}^{\nabla \mathrm{J}}$ is the isochoric part of the elasticity tensor and $\mathbb{C}_{\mathrm{vol}}^{\nabla \mathrm{J}}$ is the volumetric part. The final form of the elasticity tensor is then given by

$$
\mathbb{C}=J^{-1}\left(\overline{\mathbb{C}}^{\nabla \mathrm{J}}+\mathbb{C}_{\mathrm{vol}}^{\nabla \mathrm{J}}\right) .
$$

\subsection{Fourth-order elasticity tensor}

In this subsection we derive the fourth-order elasticity tensor of a transversely isotropic, compressible, hyperelastic material. To keep the presentation clear and concise, we reduce the number of invariants in the energy function and consider

$$
W=\bar{W}\left(\bar{I}_{1}, \bar{I}_{4}\right)+U(J),
$$

which replaces (14). From (24) we find that the expression of the Cauchy stress simplifies to

$$
\boldsymbol{\sigma}=2 J^{-1}\left[\bar{W}_{1} \operatorname{dev} \overline{\mathbf{B}}+\bar{W}_{4} \operatorname{dev}(\overline{\mathbf{m}} \otimes \overline{\mathbf{m}})\right]+\frac{\mathrm{d} U}{\mathrm{~d} J} \mathbf{I},
$$

where the terms containing derivatives with respect to $\bar{I}_{2}$ and $\bar{I}_{5}$ have been eliminated. Using the isochoric part in (66) we find that the increment of the $J \overline{\boldsymbol{\sigma}}$ has the form

$$
\begin{aligned}
\mathrm{d}(J \overline{\boldsymbol{\sigma}}) & =2\left[\mathrm{~d} \bar{W}_{1} \operatorname{dev} \overline{\mathbf{B}}+\bar{W}_{1} \mathrm{~d}(\operatorname{dev} \overline{\mathbf{B}})\right. \\
& \left.+\mathrm{d} \bar{W}_{4} \operatorname{dev}(\overline{\mathbf{m}} \otimes \overline{\mathbf{m}})+\bar{W}_{4} \mathrm{~d}(\operatorname{dev}(\overline{\mathbf{m}} \otimes \overline{\mathbf{m}}))\right] .
\end{aligned}
$$

For reference, we provide the explicit expression of the incremental components in (67), beginning with the increment of $\bar{W}_{1}$

$$
\mathrm{d} \bar{W}_{1}=\bar{W}_{11} \mathrm{~d} \bar{I}_{1}+\bar{W}_{14} \mathrm{~d} \bar{I}_{4}
$$


where $\bar{W}_{11}=\partial^{2} \bar{W} / \partial \bar{I}_{1}^{2}$ and, similarly, $\bar{W}_{14}=\partial^{2} \bar{W} / \partial \bar{I}_{1} \partial \bar{I}_{4}$. The increments of the isochoric invariants $\bar{I}_{1}$ and $\bar{I}_{4}$ are, respectively,

$$
\mathrm{d} \bar{I}_{1}=2 \overline{\mathbf{F}}: \mathrm{d} \overline{\mathbf{F}}, \quad \mathrm{d} \bar{I}_{4}=2 \mathrm{~d} \overline{\mathbf{F}} \mathbf{M} \cdot \overline{\mathbf{m}},
$$

where we have used the connection $\overline{\mathbf{m}}=\overline{\mathbf{F}} \mathbf{M}$. The increment of the isochoric deformation gradient $\mathrm{d} \overline{\mathbf{F}}$ is

$$
\mathrm{d} \overline{\mathbf{F}}=\mathrm{d} \mathbf{l} \overline{\mathbf{F}}-\frac{1}{3}(\mathbf{I}: \mathrm{d} \mathbf{d}) \overline{\mathbf{F}}=\mathrm{d} \mathbf{l} \overline{\mathbf{F}}-\frac{1}{3} \mathrm{~d} \epsilon^{\mathrm{vol}} \overline{\mathbf{F}},
$$

where $\mathrm{d} \mathbf{d}$ is the symmetric part of the incremental displacement gradient $\mathrm{d} \mathbf{l}$ defined in (56) and $\mathrm{d} \epsilon^{\mathrm{vol}}$ is the divergence of du. Substituting (69) and (70) into (68), we obtain

$$
\mathrm{d} \bar{W}_{1}=2\left[\bar{W}_{11} \operatorname{dev} \overline{\mathbf{B}}+\bar{W}_{14} \operatorname{dev}(\overline{\mathbf{m}} \otimes \overline{\mathbf{m}})\right]: \mathrm{d} \mathbf{d},
$$

where we used the property $\overline{\mathbf{B}}: \mathrm{d} \mathbf{l}=\overline{\mathbf{B}}$ : ddd. Similarly, we determine the increment of $\bar{W}_{4}$ as

$$
\begin{aligned}
\mathrm{d} \bar{W}_{4} & =\bar{W}_{14} \mathrm{~d} \bar{I}_{1}+\bar{W}_{44} \mathrm{~d} \bar{I}_{4}, \\
& =2\left[\bar{W}_{14} \operatorname{dev} \overline{\mathbf{B}}+\bar{W}_{44} \operatorname{dev}(\overline{\mathbf{m}} \otimes \overline{\mathbf{m}})\right]: \mathrm{d} \mathbf{d},
\end{aligned}
$$

where $\bar{W}_{44}=\partial^{2} \bar{W} / \partial \bar{I}_{4}^{2}$. Equation (67) includes the increment of $\operatorname{dev} \overline{\mathbf{B}}$, which is given by

$$
\mathrm{d}(\operatorname{dev} \overline{\mathbf{B}})=\mathrm{d}(\mathbb{P}: \overline{\mathbf{B}})=\mathbb{P}: \mathrm{d} \overline{\mathbf{B}}=\operatorname{dev}(\mathrm{d} \overline{\mathbf{B}}),
$$

where $\mathbb{P}$ is the fourth-order projection tensor defined in Subsection 2.2. Using equation (70) the increment of $\overline{\mathbf{B}}$ has the form

$$
\mathrm{d} \overline{\mathbf{B}}=\mathbb{H}: \mathrm{d} \mathbf{e}+\mathrm{d} \mathbf{w} \overline{\mathbf{B}}-\overline{\mathbf{B}} \mathrm{d} \mathbf{w},
$$

where the fourth-order tensor $\mathbb{H}$ is defined as $\mathbb{H}_{i j k l}=1 / 2\left(\delta_{i k} \bar{B}_{j l}+\delta_{i l} \bar{B}_{j k}+\right.$ $\left.\bar{B}_{i k} \delta_{j l}+\bar{B}_{i l} \delta_{j k}\right)$ and we introduce the increment of the deviatoric strain rate, de, defined as $\operatorname{de}=\operatorname{dev}(\mathrm{d} \mathbf{d})$. We may now write equation (73) as

$$
\mathrm{d}(\operatorname{dev} \overline{\mathbf{B}})=\operatorname{dev}(\mathbb{H}: \operatorname{de})+\mathrm{d} \mathbf{w} \overline{\mathbf{B}}-\overline{\mathbf{B}} \mathrm{d} \mathbf{w} .
$$

Following a similar process that lead to $(75)$, the increment of $[\operatorname{dev}(\overline{\mathbf{m}} \otimes \overline{\mathbf{m}})]$ is given by

$$
\begin{aligned}
\mathrm{d}[\operatorname{dev}(\overline{\mathbf{m}} \otimes \overline{\mathbf{m}})] & =\operatorname{dev}[\mathrm{d}(\overline{\mathbf{m}} \otimes \overline{\mathbf{m}})] \\
& =\operatorname{dev}(\mathbb{A}: \operatorname{de})+\mathrm{d} \mathbf{w}(\overline{\mathbf{m}} \otimes \overline{\mathbf{m}})-(\overline{\mathbf{m}} \otimes \overline{\mathbf{m}}) \mathrm{d} \mathbf{w}
\end{aligned}
$$


where $\mathbb{A}$ is the fourth-order tensor $\mathbb{A}_{i j k l}=1 / 2\left(\bar{m}_{k} \bar{m}_{j} \delta_{i l}+\bar{m}_{l} \bar{m}_{j} \delta_{i k}+\bar{m}_{i} \bar{m}_{k} \delta_{j l}+\right.$ $\bar{m}_{i} \bar{m}_{l} \delta_{j k}$ ). Substituting (71), (72), (75) and (76) into (67) gives the increment of the Kirchhoff stress, which, when compared to (62), provides the expression for the isochoric tangent stiffness related to the Jaumann rate

$$
\begin{aligned}
\overline{\mathbb{C}}^{\nabla \mathrm{J}} & =2\left[2 \left(\bar{W}_{11} \operatorname{dev} \overline{\mathbf{B}} \otimes \operatorname{dev} \overline{\mathbf{B}}+\bar{W}_{14} \operatorname{dev} \overline{\mathbf{B}} \otimes \operatorname{dev}(\overline{\mathbf{m}} \otimes \overline{\mathbf{m}})\right.\right. \\
& \left.+W_{14} \operatorname{dev}(\overline{\mathbf{m}} \otimes \overline{\mathbf{m}}) \otimes \operatorname{dev} \overline{\mathbf{B}}+\bar{W}_{44} \operatorname{dev}(\overline{\mathbf{m}} \otimes \overline{\mathbf{m}}) \otimes \operatorname{dev}(\overline{\mathbf{m}} \otimes \overline{\mathbf{m}})\right) \\
& +\bar{W}_{1}\left(\mathbb{H}-\frac{2}{3}(\mathbf{I} \otimes \overline{\mathbf{B}}+\overline{\mathbf{B}} \otimes \mathbf{I})+\frac{2}{9} \bar{I}_{1} \mathbf{I} \otimes \mathbf{I}\right) \\
& \left.+\bar{W}_{4}\left(\mathbb{A}-\frac{2}{3}(\mathbf{I} \otimes \overline{\mathbf{m}} \otimes \overline{\mathbf{m}}+\overline{\mathbf{m}} \otimes \overline{\mathbf{m}} \otimes \mathbf{I})+\frac{2}{9} \bar{I}_{4} \mathbf{I} \otimes \mathbf{I}\right)\right] .
\end{aligned}
$$

The remaining increment involves the volumetric part of the Cauchy stress, $J \boldsymbol{\sigma}_{\mathrm{vol}}$ and we write this in the form

$$
\mathrm{d}\left(J \boldsymbol{\sigma}_{\mathrm{vol}}\right)=\mathrm{d} J\left(\frac{\mathrm{d} U}{\mathrm{~d} J}+J \frac{\mathrm{d}^{2} U}{\mathrm{~d} J^{2}}\right) \mathbf{I},
$$

where the increment of $J$ is given by $\mathrm{d} J=J \mathrm{~d} \epsilon^{\mathrm{vol}}$. Comparing with (63) we find that $\mathbb{C}_{\text {vol }}^{\nabla \mathrm{J}}$ has the form

$$
\mathbb{C}_{\mathrm{vol}}^{\nabla \mathrm{J}}=J\left(\frac{\mathrm{d} U}{\mathrm{~d} J}+J \frac{\mathrm{d}^{2} U}{\mathrm{~d} J^{2}}\right) \mathbf{I} \otimes \mathbf{I}
$$

The elasticity tensor $\mathbb{C}$ of $(65)$ can now be expressed with the use of (77) and (79).

\subsection{Fourth-order pseudo-elasticity tensor}

In Section 2.4, the theory of pseudo-elasticity was introduced to account for the hysteretic behavior during loading-unloading and reloading. Specifically, equation (43) shows a pseudo-energy function where the isochoric part of the energy is scaled by $\eta$. This variable is active during unloading where, as a consequence, the deviatoric stress component is scaled with equality holding when unloading is initiated, see equation (46). Using a pseudo-elastic energy function requires some changes to the presentation in Section 3.1.

We assume that stress-softening is induced by the isochoric part of the deformation and therefore the increment of the isochoric stress component needs to be re-evaluated. This is given by

$$
\mathrm{d}\left(\eta J \overline{\boldsymbol{\sigma}}_{0}\right)=\mathrm{d} \eta\left(J \overline{\boldsymbol{\sigma}}_{0}\right)+\eta \mathrm{d}\left(J \overline{\boldsymbol{\sigma}}_{0}\right),
$$


where $\mathrm{d}\left(J \overline{\boldsymbol{\sigma}}_{0}\right)$ is given by (67). Equations (48), (49) and (50) show that $\eta$ is dependent on the deformation through $\bar{W}_{0}$, therefore the increment of $\eta$ has the form

$$
\mathrm{d} \eta=\frac{\mathrm{d} \eta}{\mathrm{d} \bar{W}_{0}} \mathrm{~d} \bar{W}_{0}
$$

The increment of the energy $\bar{W}_{0}$ is

$$
\mathrm{d} \bar{W}_{0}=\frac{\partial \bar{W}_{0}}{\partial \mathbf{F}} \mathrm{d} \mathbf{F}=(J \overline{\boldsymbol{\sigma}}): \mathrm{d} \mathbf{d}
$$

where we recall that the increment of the deformation gradient is $\mathrm{d} \mathbf{F}=\mathrm{d} \mathbf{l} \mathbf{F}$, which is different from the virtual increment defined in (51). The modified elasticity tensor $\overline{\mathbb{C}}^{\nabla \mathrm{J}}$ becomes

$$
\overline{\mathbb{C}}^{\nabla \mathrm{J}}=\frac{\mathrm{d} \eta}{\mathrm{d} \bar{W}_{0}}(J \overline{\boldsymbol{\sigma}}) \otimes(J \overline{\boldsymbol{\sigma}})+\eta \overline{\mathbb{C}}_{0}^{\nabla \mathrm{J}},
$$

where the first term accounts for the rate the material is softening and the second term gives the scaled primary response. The specific form of $\mathrm{d} \eta / \mathrm{d} \bar{W}_{0}$ depends on the definition of $\eta$, see equations (48), (49) and (50).

\subsection{A model for active-passive material}

The equations derived in Sections 3.1 and 3.2 are used to model the continuous transition between active and passive states of a muscle. Let the isochoric part of the energy function (65) be given by the sum of two contributions such that

$$
W=\bar{W}_{\text {iso }}\left(\bar{I}_{1}\right)+\bar{W}_{\text {fib }}\left(\bar{I}_{4}\right)+U(J),
$$

where $\bar{W}_{\text {iso }}\left(\bar{I}_{1}\right)$ accounts for the isotropic base matrix consisting of connective tissue and $\bar{W}_{\text {fib }}\left(\bar{I}_{4}\right)$ for the oriented fibers in the active or passive state. The isotropic contribution is given by the volume-preserving neo-Hookean model

$$
\bar{W}_{\text {iso }}\left(\bar{I}_{1}\right)=\frac{\mu}{2}\left(\bar{I}_{1}-3\right),
$$

where $\mu$ is the shear modulus in the undeformed configuration. The fiber reinforcement is taken to be the sum of an active and passive potential in the form

$$
\bar{W}_{\text {fib }}\left(\bar{I}_{4}\right)=(1-\alpha) \bar{W}_{\text {pass }}\left(\bar{I}_{4}\right)+\alpha \bar{W}_{\text {act }}\left(\bar{I}_{4}\right),
$$


where $\alpha$ is a scalar representing the volume ratio of active fibers, similar to the approach taken by Blemker et al. (2003); Odegard et al. (2008); Parente et al. (2009); Shim et al. (2012). The value of $\alpha$ is independent of the deformation and is defined by the relation

$$
\alpha(s)=\tanh \left(\frac{s-\hat{s}_{i}}{k}\right),
$$

where $\hat{s}_{\mathrm{i}}$ is the value of $s$ at the instant when a stimulus is administrated to the passive muscle and $k$ is a material parameter governing the rate of change between states. We recall that the variable $s$ has been introduced in Section 2.3 to identify all possible configurations $\mathcal{B}(s)$ of the body.

The energy functions $\bar{W}_{\text {pass }}\left(\bar{I}_{4}\right)$ and $\bar{W}_{\text {act }}\left(\bar{I}_{4}\right)$ are taken as

$$
\bar{W}_{\text {pass }}\left(\bar{I}_{4}\right)=\frac{\mu \mu_{\mathrm{p}}}{2}\left(\bar{I}_{4}-1\right)^{2}, \quad \bar{W}_{\text {act }}\left(\bar{I}_{4}\right)=\frac{\mu \mu_{\mathrm{a}}}{2}\left(\hat{\bar{I}}_{4}-\bar{I}_{4_{0}}\right)^{2},
$$

where $\bar{W}_{\text {pass }}\left(\bar{I}_{4}\right)$ is the standard reinforcing model with $\mu_{\mathrm{p}}$ as a dimensionless parameter providing the degree of anisotropy due to fibers in the passive state (Horgan and Saccomandi, 2005; Merodio and Ogden, 2005; Qiu and Pence, 1997). The formulation of $\bar{W}_{\text {act }}\left(\bar{I}_{4}\right)$ is similar, however $\bar{W}_{\text {act }}=0$ when the stretch of the active fiber $\hat{\bar{I}}_{4}$ is equal to its rest length $\bar{I}_{4_{0}}$. The parameter $\mu_{\mathrm{a}}$ is again dimensionless and accounts for the anisotropy provided by the active fibers. It dependence on the deformation as

$$
\mu_{\mathrm{a}}=c_{1} \mathrm{e}^{-\left(\hat{\bar{I}}_{4}-I_{4}\right) / c_{2}},
$$

where $c_{1}$ and $c_{2}$ are determined from experimental data. The sign of the exponent accounts for the decrease in actomyosin overlap as the fiber stretches reducing the magnitude of $\mu_{\mathrm{a}}$, see Paetsch et al. (2012).

Finally, the volumetric portion of the strain energy is taken as the penalty function

$$
U(J)=\frac{\kappa}{2}(J-1)^{2},
$$

where $\kappa$ is known as the penalty parameter (Prot and Skallerud, 2009). For nearly incompressible materials, $\kappa$ is taken to be sufficiently large to minimize change in volume.

Using the components of the energy function in (85), (86) and (88), we now determine the derivatives of $W$ to calculate the Cauchy stress (66) and 
the components of the elasticity and pseudo-elasticity tensors (79) and (83), respectively. Note that the decoupled form (84) results in $\bar{W}_{14}=0$.

The first- and second-order derivative of $W$ with respect to $\bar{I}_{1}$ have the forms

$$
\bar{W}_{1}=\frac{\mu}{2}, \quad \bar{W}_{11}=0,
$$

and those with respect to $\bar{I}_{4}$ are

$$
\bar{W}_{4}=(1-\alpha) \bar{W}_{\text {pass }}^{\prime}+\alpha \bar{W}_{\text {act }}^{\prime}, \quad \bar{W}_{44}=(1-\alpha) \bar{W}_{\text {pass }}^{\prime \prime}+\alpha \bar{W}_{\text {act }}^{\prime \prime},
$$

where

$$
\begin{aligned}
& \bar{W}_{\text {pass }}^{\prime}=\mu \mu_{\mathrm{p}}\left(\bar{I}_{4}-1\right), \\
& \bar{W}_{\text {act }}^{\prime}=\frac{\mu \mu_{\mathrm{a}}^{\prime}}{2}\left(\hat{\bar{I}}_{4}-\bar{I}_{4_{0}}\right)^{2}+\mu \mu_{\mathrm{a}} \bar{I}_{4_{0}}^{-1}\left(\hat{\bar{I}}_{4}-\bar{I}_{4_{0}}\right), \\
& \bar{W}_{\text {pass }}^{\prime \prime}=\mu \mu_{\mathrm{p}}, \\
& \bar{W}_{\text {act }}^{\prime \prime}=\frac{\mu \mu_{\mathrm{a}}^{\prime \prime}}{2}\left(\hat{\bar{I}}_{4}-\bar{I}_{4_{0}}\right)^{2}+2 \mu \mu_{\mathrm{a}}^{\prime} \bar{I}_{4_{0}}^{-1}\left(\hat{\bar{I}}_{4}-\bar{I}_{4_{0}}\right)+\mu \mu_{\mathrm{a}} \bar{I}_{4_{0}}^{-2},
\end{aligned}
$$

and

$$
\mu_{\mathrm{a}}^{\prime}=-\frac{c_{1}}{c_{2}} \bar{I}_{4_{0}}^{-1} \mathrm{e}^{-\left(\hat{\bar{I}}_{4}-\bar{I}_{4_{0}}\right) / c_{2}}, \quad \mu_{\mathrm{a}}^{\prime \prime}=\frac{c_{1}}{c_{2}^{2}} \bar{I}_{4_{0}}^{-2} \mathrm{e}^{-\left(\hat{\bar{I}}_{4}-\bar{I}_{4_{0}}\right) / c_{2}} .
$$

The volumetric part of the energy function $U(J)$ has the derivatives

$$
\frac{\mathrm{d} U}{\mathrm{~d} J}=\kappa(J-1), \quad \frac{\mathrm{d}^{2} U}{\mathrm{~d} J^{2}}=\kappa .
$$

An expression for the Cauchy stress is obtained by substituting $(91)_{1},(92)_{1}$ and (98) ${ }_{1}$ into (66). The derivatives of $W$ given by $(91)_{1},(92)$ and (98) are needed in (79) and (83) to obtain $\mathbb{C}_{\mathrm{vol}}^{\nabla J}$ and $\overline{\mathbb{C}}^{\nabla J}$, respectively. The required fourth-order pseudo-elasticity tensor is then obtained from (64).

\section{Numerical results}

We present numerical results to validate and verify the development of the previous sections. The numerical simulations are carried out in Abaqus/Standard, where the expressions of the Cauchy stress and the fourth-order pseudoelasticity tensor are defined in the subroutine UMAT (Simulia, 2009).

Experimental data have been used to determine material model parameters by Paetsch et al. (2012) and the same values are used for the numerical 
simulations in this section. The additional parameter $\kappa$, used to define the volumetric portion of the energy (90), is chosen to be appropriately large to ensure nearly incompressible behavior. For reference, the material model parameters and their values are listed in Table 1.

The intermediate configuration $\hat{\mathcal{B}}$, occupied by the active muscle in the absence of applied boundary traction, has been defined in Section 2.3 to depend on muscle physiology and structure. We therefore assume, for simplicity, that the resulting isochoric deformation gradient tensor $\overline{\mathbf{F}}_{0}$ has the diagonal components

$$
\lambda_{0_{1}}=0.95, \quad \lambda_{0_{2}}=\lambda_{0_{3}}=\lambda_{0_{1}}^{-1 / 2} .
$$

Table 1: Summary of material model parameters describing the passive and active states and the continuous transition. The units of parameters are noted with the others being dimensionless.

\begin{tabular}{lcccccccccc}
\hline \hline & $\mu$ & $\mu_{p}$ & $\kappa$ & $c_{1}$ & $c_{2}$ & $r$ & $m$ & $a$ & $b$ & $k$ \\
& $(\mathrm{~Pa})$ & - & $(\mathrm{Pa})$ & - & - & - & $(\mathrm{kPa})$ & $(\mathrm{kPa})$ & $(\mathrm{kPa})$ & - \\
\hline passive & 755.5 & 86.13 & $2.0 \mathrm{e} 9$ & 470.0 & 0.95 & 1.05 & 2.5 & 0.1 & 2.5 & 0.2 \\
active & 755.5 & 86.13 & $2.0 \mathrm{e} 9$ & 470.0 & 0.95 & 1.55 & 30.0 & 30.0 & 15.0 & 0.2 \\
\hline \hline
\end{tabular}

\subsection{Uniaxial extension}

To illustrate the theory we consider first the simple situation in which a muscle is subject to two consecutive loading-unloading cycles with uniaxial tension in the fiber direction with principal Cauchy stress components given by $\sigma_{1}=\sigma$ and $\sigma_{2}=\sigma_{3}=0$. The results of this simulation are used to validate the model by comparing to the experimental data and to the exact solution given by Paetsch et al. (2012).

More particularly, the applied maximum extension in the 1-direction is $\lambda_{1}=\lambda=1.15$ and the material is free to contract in the other two directions. Figure 1(a) shows the muscle subject to two loading-unloading cycles. During the first unloading, the muscle is activated and allowed to transition from passive to active state. The numerical results show the effects of stress softening during both cycles as the unloading paths does not follow the loading paths, regardless of activation state. Furthermore, it can be 
seen that the material returns to the reference length. However, in this configuration the muscle is no longer stress-free due to the transition between the passive to the active state. In Figure 1(b) we show the change in energy as given by (43). Note the increase in energy during the first unloading when the muscle transitions to the active state. The value of the pseudoenergy when the muscle is returned to the reference configuration, i.e. the value of $\phi(\eta)$ at $\lambda=1$, represents the cumulative energy dissipated during the deformation cycle. The results presented in Figure 1 essentially coincide with the analytical response and closely approximate the experimental data shown by Paetsch et al. (2012). There exists a very small difference between the analytical and numerical results due to the incompressibility constraint being enforced by a penalty expression in the energy function.

(a)

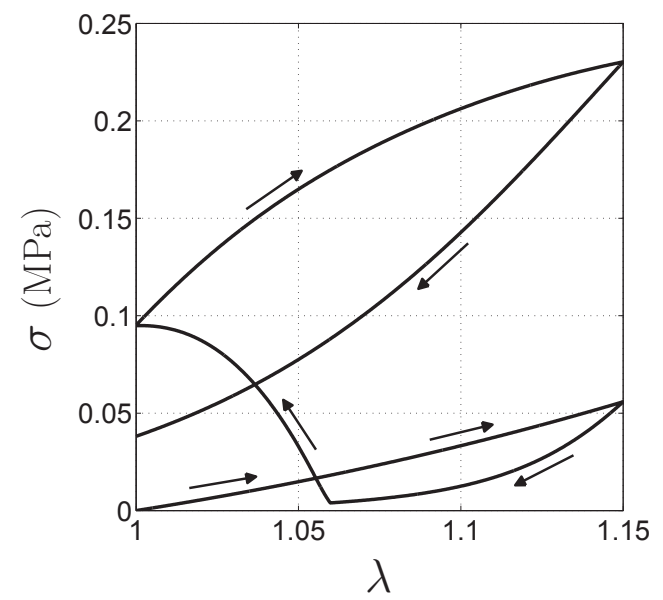

(b)

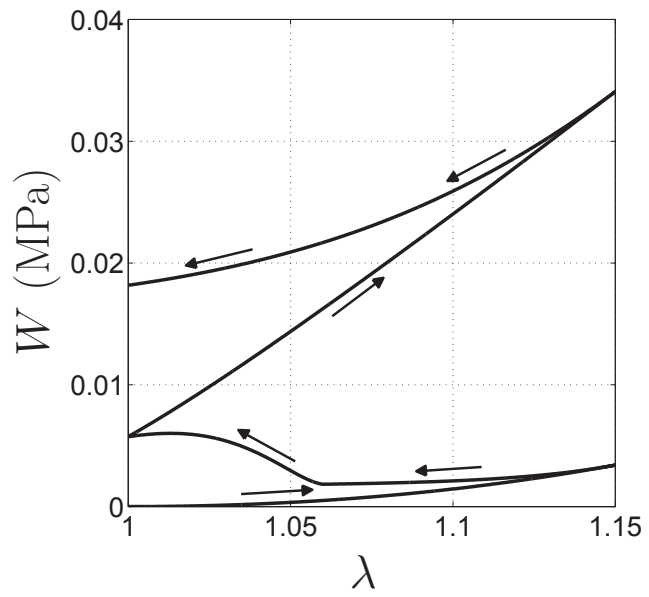

Figure 1: Numerical results produced by Abaqus show the response to uniaxial tension subject to a stimulus during the first unloading with a) the Cauchy stress of the muscle and $b$ ) the amount of energy stored and returned during cyclic loading and unloading.

\subsection{Biohybrid thin film}

In this section we consider a biohybrid thin film consisting of synthetic polymer and muscular substrates for potential use in soft robotics. The review of recent experimental results of biohybrid thin films and soft robotic actuators is outside the scope of this study and we refer to the publications 
by Feinberg et al. (2007); Alford et al. (2010); Shim et al. (2012); Nawroth et al. (2012); Baryshyan et al. (2012) for details. For example, Feinberg et al. (2007) and Nawroth et al. (2012), report the use of rat neonatal cardiomyocytes on a thin polymer substrate to build muscular thin films for bio-inspired complex movements such as gripping and swimming. In particular, in (Feinberg et al., 2007) rectangular strips are cut from the thin film with the muscle fibers oriented parallel to the longest dimension. It is shown that cyclic contractions of the muscle fibers induces a continuous transformation of the rectangular strip from an uncoiled to a coiled state.

The model will now be used to simulate experimental results reported in (Feinberg et al., 2007). Figure 2 shows the geometric layout of the rectangular strip that will be used to determine the movement of a biohybrid gripper. The strip, in its reference configuration $\mathcal{B}_{0}$, has a uniform thickness with top and bottom faces normal to the $X_{1}$ direction and dimensions of $1 \mathrm{~mm}$ and $10 \mathrm{~mm}$ in the $X_{2}$ and $X_{3}$ directions, respectively, where we recall that $\left(X_{1}, X_{2}, X_{3}\right)$ are reference rectangular Cartesian coordinates. The total thickness in $\mathcal{B}_{0}$ is equal to $55 \mu \mathrm{m}$, the substrate of the muscular tissue is $15 \mu \mathrm{m}$, the layer of the synthetic polymer is $45 \mu \mathrm{m}$. Before activation, all fibers are parallel and oriented along the rectangular length.

The synthetic polymer is considered hyperelastic with shear modulus equal to $100 \mathrm{kPa}$, bulk modulus equal to $2 \mathrm{GPa}$ and stress-stretch relation given by the neo-Hookean model. The muscular substrate is initially in the passive state. During activation the material deforms and the strip is subject to a continuous transformation as shown in Figure 3. The value $\alpha$ represents the volume ratio of active muscles and for $\alpha=0.90$ the initially flat strip has turn into circular shaped ring. At this point the stimulation of the muscle is terminated and the tissue transitions to the passive state. For $\alpha=0.0$ the thin film returns to the initial geometry.

\section{Conclusion}

This paper presents a generalization of the constitutive law presented by Paetsch et al. (2012) to simulate muscle transitioning between active and passive states. The material considered is pseudo-elastic, nearly incompressible and transversely isotropic with fiber orientation parallel to the longitudinal direction of the muscle. To account for fiber activation we use the multiplicative decomposition of the deformation gradient and define stress-free configurations for both the active and passive states. 


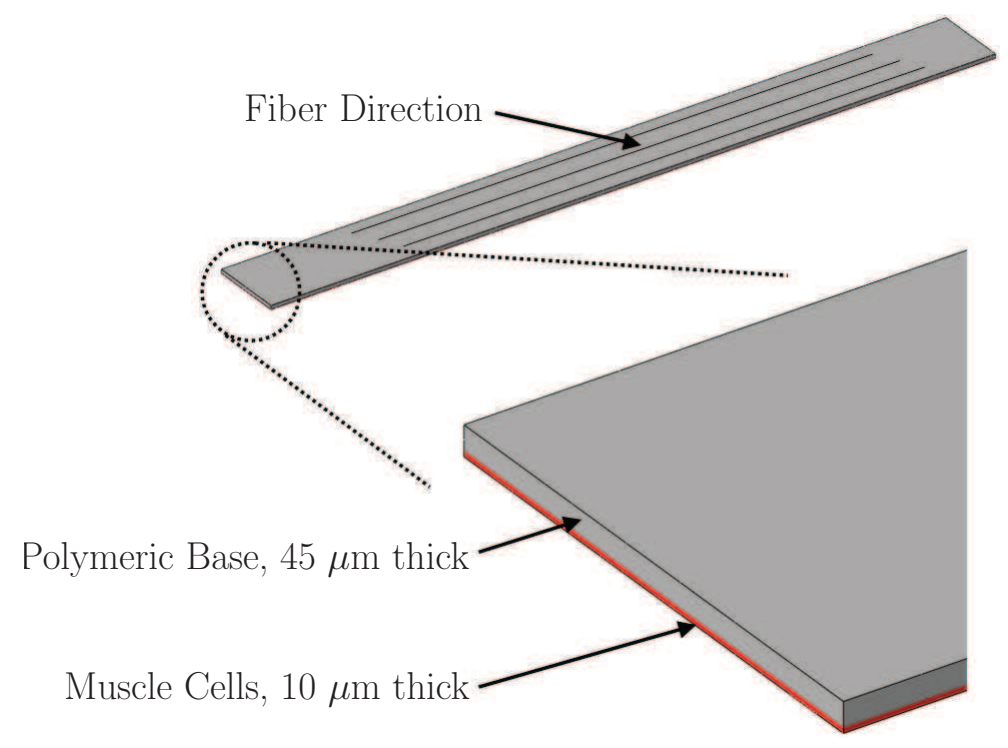

Figure 2: The bioactuator model includes a $45 \mu \mathrm{m}$ polymeric base layer on top of a thinner $10 \mu \mathrm{m}$ layer of muscle tissue with fibers aligned parallel to the rectangular length.

The simple case of cyclic loading in uniaxial tension demonstrate the characteristics of the model. The analysis illustrates the effect of muscle stimulation as stress increases with decreasing strain while transitioning between passive and active states. The material is no longer stress-free when returning to the undeformed state. The results also show that the loading and unloading paths do not coincide, indicating energy dissipation. A second example, based on experimental data of muscular tissue, shows the behavior of Manduca muscle in active biohybrid constructs.

The ability to analyze the response of active biohybrid material with complex geometries under non-trivial boundary conditions can aid in the development of future devices.

\section{Acknowledgments}

This publication was based on work supported in part by Award No KUKC1-013-04, made by King Abdullah University of Science and Technology (KAUST). The work of CP was supported in part by the National Science Foundation IGERT Grant DGE-1144591. 

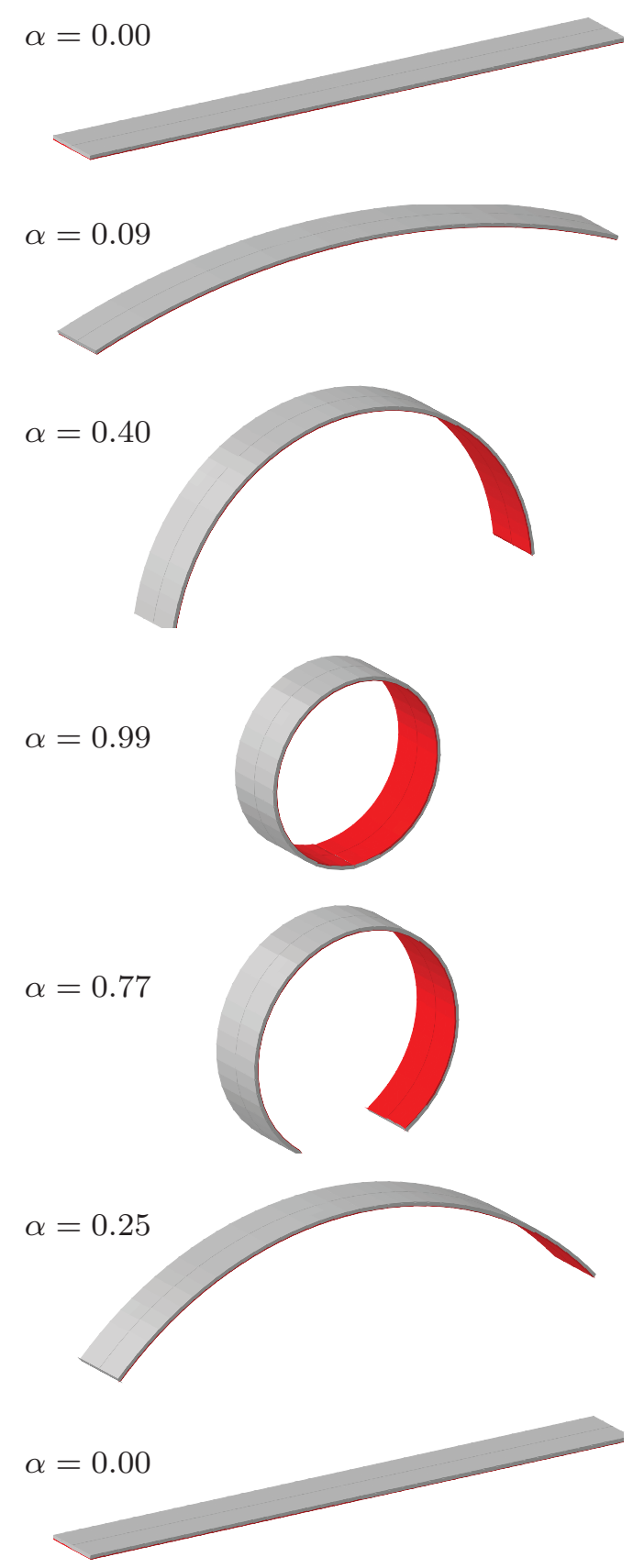

Figure 3: Model results show a biohybrid thin film transitioning between passive and active states. Contraction of the muscle fibers induces a continuous transformation of the rectangular strip from an uncoiled to a coiled configuration and vice versa. 


\section{References}

Akiyama, Y., Iwabuchi, K., Furukawa, Y., Morishima, K., 2010. Electrical stimulation of cultured lepidopteran dorsal vessel tissue: an experiment for development of bioactuators. In Vitro Cell. Dev. - An. 46, 411-415.

Alford, P. W., Feinberg, A. W., Sheehy, S. P., Parker, K. K., 2010. Biohybrid thin films for measuring contractility in engineered cardiovascular muscle. Biomaterials 31, 3613-3621.

Ambrosi, D., Pezzuto, S., 2012. Active stress vs. active strain in mechanobiology: Constitutive issues. J. Elasticity 107, 199-212.

Baryshyan, A. L., Woods, W., Trimmer, B. A., Kaplan, D. L., 2012. Isolation and maintenance-free culture of contractile myotubes from Manduca sexta embryos. PLoS ONE 7, e31598.

Blemker, S. S., Pinsky, P. M., Delp, S. L., 2003. A 3d model of muscle reveals the causes of nonuniform strains in the biceps. J. Biomech. 358, 657-665.

Boel, M., Reese, S., Parker, K. K., Kuhl, E., 2009. Computational modeling of muscular thin films for cardiac repair. Comput. Mech. 43 (4), 535-544.

Bose, K., Dorfmann, A., 2009. Computational aspects of a pseudo-elastic constitutive model for muscle properties in a soft-bodied arthropod. Int. J. Solids Struct. 44, 42-50.

Cherubini, C., Filippi, S., Nardinocchi, P., Teresi, L., 2008. An electromechanical model of cardiac tissue: constitutive issues and electrophysiological effects. Prog. Biophys. Mol. Bio. 97, 562-573.

Craig, R., Padrón, R., 2004. Molecular structure of the sarcomere. McGrawHill.

Demirkoparan, H., Pence, T. J., Wineman, A., 2009. On dissolution and reassembly of filamentary reinforcing networks in hyperelastic materials. Proc. R. Soc. London, Ser. A. 465, 867-894.

Dorfmann, A., Ogden, R. W., 2003. A pseudo-elastic model for loading, partial unloading and reloading of particle-reinforced rubber. Int. J. Solids Struct. 40, 2699-2714. 
Dorfmann, A., Ogden, R. W., 2004. A constitutive model for the Mullins effect with permanent set in particle-reinforced rubber. Int. J. Solids Struct. 41, 1855-1878.

Dorfmann, A., Trimmer, B. A., Woods, W. A., 2007. A constitutive model for muscle properties in a soft-bodied arthropod. J. Roy. Soc. Interface 4, $257-269$.

Dorfmann, A. L., Woods, W. A., Trimmer, B. A., 2008. Muscle performance in a soft-bodied terrestrial crawler: constitutive modeling of strain-rate dependency. J. Roy. Soc. Interface 5, 349-362.

Ehret, A. E., Böl, M., Itskov, M., 2011. A continuum constitutive model for the active behavior of skeletal muscle. J. Mech. Phys. Solids 59, 625-636.

Federico, S., Gasser, T. C., 2010. Nonlinear elasticity of biological tissues with statistical fibre orientation. J. Roy. Soc. Interface 7, 955-966.

Feinberg, A. W., Feigel, A., Shevkoplyas, S. S., Sheehy, S., Whitesides, G. M., Parker, K. K., 2007. Muscular thin films for building actuators and powering devices. Science 317, 1366-1370.

Flory, P. J., 1961. Thermodynamic relations for highly elastic materials. Trans. Faraday Soc. 57, 829-838.

Fujita, H., Dau, V. T., Shimizu, K., Hatsuda, R., Sugiyama, S., Nagamori, E., 2011. Designing of a Si-MEMS device with an integrated skeletal muscle cell-based bio-actuator. Biomed. Microdevices. 13, 123-129.

Fung, Y. C., 1967. Elasticity of soft tissues in simple elongation. Am. J. Physiol. 213, 1532-1544.

Fung, Y. C., 1980. On pseudo-elasticity of living tissues. In: Nasser, S. N. (Ed.), Mechanics today. Vol. 5 of Pergamon mechanics today series. Pergamon Press, New York, NY, pp. 49-66.

Herr, H., Dennis, R., 2004. A swimming robot actuated by living muscle tissue. J. Neuroeng. Rehabil. 1, 1-9.

Hill, A. V., 1938. The heat of shortening and dynamic constants of muscle. Proc. R. Soc. London B 126, 136-195. 
Hollerbach, J. M., Hunter, I. W., Ballantyne, J., 1991. A comparative analysis of actuator technologies for robotics. In: O. Khatib, J. C., Lozano-Perez, T. (Eds.), The Robotics Review. MIT Press, pp. 301-342.

Holzapfel, G. A., 2000. Nonlinear Solid Mechanics: A Continuum Approach for Engineering. John Wiley \& Sons, Chichester.

Horgan, C. O., Ogden, R. W., Saccomandi, G., 2004. A theory of stress softening of elastomers based on finite chain extensibility. P. Roy. Soc. A-Math. Phy. 460, 1737-1754.

Horgan, C. O., Saccomandi, G., 2005. A new constitutive theory for fiberreinforced incompressible nonlinearly elastic solids. J. Mech. Phys. Solid $53,1985-2015$.

Ito, D., Tanaka, E., Yamamoto, S., 2010. A novel constitutive model of skeletal muscle taking into account anisotropic damage. J. Mech. Behavior Biomed. Mat. 3, 85-93.

Lieber, R. L., 2002. Skeletal Muscle Structure, Function, \& Plasticity. Lippincott Williams \& Wilkins, Baltimore, MD.

Merodio, J., Ogden, R. W., 2005. Mechanical response of fiber-reinforced incompressible non-linear elastic solids. Int. J. Nonlinear Mech. 40, 213227.

Nardinocchi, P., Teresi, L., 2007. On the active response of soft living tissues. J. Elasticity 88, 27-39.

Nash, M. P., Hunter, P. J., 2000. Computational mechanics of the heart from tissue structure to ventricular function. J. Elasticity 61, 113-141.

Nawroth, J. C., Lee, H., Feinberg, A. W., Ripplinger, C. M., McCain, M. L., Grosberg, A., Dabiri, J. O., Parker, K. K., 2012. A tissue-engineered jellyfish with biomimetic propulsion. Nat. Biotechnol. 30, 792-797.

Nordsetten, D. A., Niederer, S. A., Nash, M. P., Hunter, P. J., Smith, N. P., 2011. Coupling multi-physics models to cardiac mechanics. Prog. Biophys. Mol. Bio. 104, 77-88.

Nossal, R., Lecar, H., 1991. Molecular and Cell Biophysics. Addison-Wesley Publishing. 
Odegard, G. M., Haut Donahue, T. L., Morrow, D. A., Kaufman, K. R., 2008. Constitutive modeling of skeletal muscle tissue with an explicit strainenergy function. J. Biomech. Eng.-Trans. ASME 130.

Ogden, R. W., 1976. Volume changes associated with the deformation of rubber-like solids. J. Mech. Phys. Solids 24, 323-338.

Ogden, R. W., 1978. Nearly isochoric elastic deformations: application to rubberlike solids. J. Mech. Phys. Solids 26, 37-57.

Ogden, R. W., 1997. Non-Linear Elastic Deformations. Dover Publications.

Ogden, R. W., 2001. Elements of the theory of finite elasticity. In: Fu, Y. B., Ogden, R. W. (Eds.), Nonlinear Elasticity: Theory and Applications. Cambridge University Press, pp. 1-58.

Ogden, R. W., Roxburgh, D. G., 1999. A pseudo-elastic model for the Mullins effect in filled rubber. P. Roy. Soc. A-Math. Phy. 455, 2861-2877.

Paetsch, C., Trimmer, B. A., Dorfmann, A., 2012. A constitutive model for active-passive transition of muscle fibers. Int. J. Nonlinear Mech. 47, 377387.

Parente, M. P. L., Natal Jorge, R. M., Mascarenhas, T., Fernandes, A. A., Martins, J. A. C., 2009. The influence of the material properties on the biomechanical behavior of the pelvic floor muscles during vaginal delivery. J. Biomech. 42, 1301-1306.

Pathmanathan, P., Chapman, S. J., Gavaghan, D. J., Whiteley, J. P., 2010. Cardiac electromechanics: the effect of contraction model on the mathematical problem and accuracy of the numerical scheme. Q. J. Mech. Appl. Math. 63, 375-399.

Perry, S. V., 1996. Molecular Mechanisms in Straited Muscle. Cambridge University Press.

Prot, V. E., Skallerud, B. H., 2009. Nonlinear solid finite element analysis of mitral valves with heterogeneous leaflet layers. Comput. Mech. 43, 353368. 
Qiu, G. Y., Pence, T. J., 1997. Remarks on the behavior of simple directionally reinforced incompressible nonlinearly elastic solids. J. Elasticity 49, $1-30$.

Rajagopal, K. R., Wineman, A. S., 1992. A constitutive equation for nonlinear solids which undergo deformation induced microstructural changes. Int. J. Plasticity 8, 385-395.

Shim, J., Grosberg, A., Nawroth, J. C., Parker, K. K., Bertoldi, K., 2012. Modeling of cardiac muscle thin films: pre-stretch, passive and active behavior. J. Biomech. 45, 832-841.

Simulia, D. S., 2009. Abaqus 6.9. Providence RI, USA.

Spencer, A. J. M., 1971. Theory of invariants. In: Eringen, A. C. (Ed.), Continuum Physics. Vol. 1. Academic Press, New York, pp. 239-353.

Stålhand, J., Klarbring, A., Holzapfel, G. A., 2011. A mechanochemical 3D continuum model for smooth muscle contraction under finite strains. J. Theor. Biol. 268, 120-130.

Tanaka, Y., Sato, K., Shimizu, T., Yamato, M., Okano, T., Kitamori, T., 2007. A micro-spherical heart pump powered by cultured cardiomyocytes. Lab Chip 7, 207-212.

Usyk, T. P., Mazhari, R., McCulloch, A. D., 2000. Effect of laminar orthotropic myofiber architecture on regional stress and strain in the canine left ventricle. J. Elasticity 61, 143-164.

Whiteley, J. P., Bishop, M. J., Gavaghan, D. J., 2007. Soft tissue modelling of cardiac fibres for use in coupled mechano-electric simulations. B. Math. Biol. 69, 2199-2225.

Woods, Jr., W. A., Fusillo, S. J., Trimmer, B. A., 2008. Dynamic properties of a locomotory muscle of the tobacco hornworm manduca sexta during strain cycling and simulated natural crawling. J. Exp. Biol. 211, 873-882.

Xi, J., Schmidt, J. J., Montemagno, C. D., 2005. Self-assembled microdevices driven by muscle. Nature Materials 4, 180-184. 



\section{RECENT REPORTS}

12/83 Object-Oriented Paradigms for Modelling Vascular Tumour Growth: a Case Study

Connor

Cooper

Byrne

Maini

McKeever

12/84 Chaste: an open source $C_{++}$library for computational physiology and biology

Mirams

Arthurs
Bernabeu

Bordas

Cooper

Corrias

Davit

Dunn

Fletcher

Harvey

Marsh

Osborne

Pathmanathan

Pitt-Francis

Southern

Zemzemi

Gavaghan

Schlackow

Marguerat

Proudfoot

Bähler

Erban

Gullerova

Lovrics

Gao

Juhász

Bock

Byrne

Dinnyés

Kovács

Taroni

Breward

Cummings

Griffiths

Morgan

Breward

Griffiths

Howell

Penfold

Thomas

Tucker

Petkov

Webster

Vigolo Griffiths 
12/97 Inference of the genetic network regulating lateral root initiation in Arabidopsis thaliana

Muraro

Voß

Wilson

Bennett

Byrne

De Smet

Hodgman

King

12/98 Axisymmetric bifurcations of thick spherical shells under inflation

deBotton and compression

Bustamante

Dorfmann

12/99 Calculus from the past: Multiple Delay Systems arising in Cancer

Wake

Cell Modelling

Byrne

12/100 Nonlocal models of electrical propagation in cardiac tissue: elec-

trotonic effects and the modulated dispersion of repolarization

Bueno-Orovio

Kay

Grau

Rodriguez

Burrage

12/101 Microfluidic Immunomagnetic Multi-Target Sorting A Model for

Tsai

Controlling Deflection of Paramagnetic Beads

Griffiths

Stone

12/102 A New Lattice Boltzmann Equation to Simulate Density-Driven

Allen Convection of Carbon Dioxide

Reis

Sun

12/103 Control and optimization of solute transport in a porous tube

Griffiths

Howell

Shipley

12/104 Air-cushioning in impact problems

Moore

Ockendon

Oliver

12/105 Strain controlled biaxial stretch: An experimental characterization

Pancheri of natural rubber

Dorfmann

Copies of these, and any other OCCAM reports can be obtained from:

Oxford Centre for Collaborative Applied Mathematics

Mathematical Institute

24 - 29 St Giles'

Oxford

OX13LB

England

www.maths.ox.ac.uk/occam 